\title{
Ten years on ART - where to now?
}

Next year marks the tenth anniversary since the rollout of antiretroviral therapy (ART) in South Africa (SA), the country with the largest number of people living with HIV in the world. SA also has the world's largest antiretroviral therapy (ART) programme, with approximately 1.8 million people estimated to have received ART by mid-2011. ${ }^{[1}$

The massive investments in treatment and prevention have been accompanied by great successes. Increasing ART coverage has led to a decline in HIV-related adult mortality. During the first 3 years of ART rollout, although ART uptake averaged around $40 \%$ of those in need, it has been estimated that AIDS mortality dropped by approximately $25 \%{ }^{[2]}$

Since the announcement of a comprehensive care, management and treatment programme by the Department of Health in late 2003, access to ART in SA has increased dramatically. Over time, revisions to the national treatment guidelines have meant that more patients than ever are eligible to initiate lifesaving therapy. The shift from an ART eligibility criterion of a CD4 count $<350$ cells/ $\mu$ l, from the previous threshold of $<200$ cells/ $\mu$, resulted in adult ART coverage dropping from $79 \%$ to $52 \%$, although clearly more patients were eligible to start ART. Although HIV testing figures have increased in the country, mainly because of the National Testing Campaign launched by the Minister of Heath in 2010, they are not yet high enough. Over a quarter of the population has been tested for HIV, but this has not translated into all those with a positive result being enrolled into care. The 2012 SA Clinicians Guideline recommends starting ART at a CD4 count $<350$ cells/ $\mu$ l and argues for the use of ART in discordant couples. This again increases the number of patients deserving of being enrolled into care.

There remain significant obstacles, both to access to ART for those who need it and to sustaining those already on treatment. The disparity between rural and more highly resourced urban areas, significant operational problems, and lack of public healthcare infrastructure and adequately trained healthcare workers to provide long-term quality care and treatment for the masses of HIV-infected patients are just some of the challenges. These obstacles must be dealt with even in the current context of severe shortages in the healthcare workforce. Two of these issues are addressed by research presented in this issue of SAMJ..$^{[3,4]}$ 
Task shifting has been widely promoted as a mechanism for expanding ART access. Changes in treatment guidelines calling for less toxic treatment regimens, higher CD4 count thresholds for initiation and simplified laboratory monitoring schedules should facilitate such task shifting to lower-level personnel. ${ }^{[5]}$ In the early years following ART rollout, Médicins Sans Frontières used task shifting in their community-based HIV programme to integrate HIV treatment successfully into well-established primary healthcare services. Strategies to increase treatment access while conserving resources without compromising patient outcomes have shown promise. Early studies of nurse-provided ART from rural Rwanda, Ethiopia and Lesotho established successful implementation. Results from the CIPRA-SA study, an SA randomised trial, confirmed that doctor-initiated, nurse-managed HIV treatment was not inferior to doctor-initiated, doctor-managed care. ${ }^{[6]}$ Long and colleagues demonstrated also that stable patients managed at primary health clinics had a similar, or even better, 12-month outcome compared with those maintained at a hospital-based ART clinic. ${ }^{[7]}$ There was the additional advantage of an $11 \%$ cost saving per patient treated. While this was not full Nurse-Initiated Management of Antiretroviral Treatment (NIMART), it did demonstrate that nurses could play a substantive role in managing HIV treatment.

Large-scale NIMART also appears to be feasible and acceptable in the primary sector health services in SA. Evidence for NIMART in Africa is limited and little is known about the key barriers to implementing these programmes on a large scale. ${ }^{[5]}$ Data from the STRETCH study (Streamlining Tasks and Roles to Expand Treatment and Care for HIV) suggest that expansion of the primary care nurse role to include ART initiation and maintenance can be done safely and will improve health outcomes and quality of care, although it may not reduce time to ART or impact on mortality. Time to death for those receiving ART from primary care nurses was similar to that in patients receiving ART from doctors (hazard ratio 0.94, 95\% confidence interval $0.76-1.15) \cdot{ }^{[5,8]}$ As Nyasulu and colleagues highlight in this issue, decentralisation of ART by primary healthcare workers increases ART uptake and reduces workload at referral facilities. ${ }^{[3]}$ In their study, 45 professional nurses from 17 primary healthcare facilities around Johannesburg were trained to initiate patients on ART. The number of initiations at the PHC facility increased significantly by 99 patients immediately after NIMART rollout and continued to increase by a further 9 initiations every month. Their study showed that decentralisation of services strengthened retention in care and reduced the burden of managing complicated cases at referral hospitals.

Although the shift to nurse-initiated care has already begun, it does have its limitations, the main ones being adequate and sustainable training, support and salaries for staff in new roles, the integration of new members into healthcare teams, and compliance with regulatory bodies. ${ }^{[9]}$ While NIMART is promising, its success will require a comprehensive approach with clinical guidelines tailored to nurses along with a strong monitoring and evaluation capacity. Accordingly, efforts to determine whether ART can be managed safely and effectively at even lower levels of the healthcare system must also be explored. We await treatment outcome data to determine whether NIMART implementation will mirror the CIPRA results.

Another problem that the SA HIV programme faces is how to deal with the twin epidemics of HIV and tuberculosis (TB). It is estimated that one-third of the people living with HIV and AIDS worldwide are co-infected with TB. SA has one of the highest estimated TB rates in the world, ranking 4 th among the 22 high-burden countries. For many years, TB and HIV care were largely separate; however, efforts to integrate TB and HIV services are included in the National Strategic Plan and National TB Programme to ensure that co-infected patients receive appropriate care and treatment. As Chimbindi and colleagues demonstrate in this issue, an integrated service that includes HIV and TB testing and treatment is convenient for most patients and ensures timely treatment initiation and optimal care..$^{[4]}$ As highlighted in this article, patients with TB face several obstacles in accessing care. However, among those who did access TB care there was a high uptake of HIV testing and appropriate management of co-infected patients in this rural public programme.

Should SA follow Ethiopia, Malawi and Uganda ${ }^{[10]}$ and consider community health worker (CHW) programmes for HIV care and treatment? CHWs could play an important role in scaling up ART by taking over a number of tasks from professional health workers. CHW programmes throughout sub-Saharan Africa have improved access to, and coverage of, communities with basic health services. There is some evidence that these programmes can improve outcomes; however, moving HIV care to CHWs has only just begun to be explored. Interestingly, findings from a community-based programme in Kenya demonstrated that task shifting of ART from healthcare workers to people living with AIDS in the community had similar outcomes in terms of detectable viral load, mean CD4 count, decline in Karnofsky score, change in ART regimen, new opportunistic infections and pregnancy rates compared with those receiving clinic-based HIV care. ${ }^{[11]}$ Evidence in areas other than HIV suggests that CHW programmes are feasible and effective. In Pakistan, community case management with oral amoxicillin of children with severe pneumonia by female health workers was equivalent to the standard of care, ${ }^{[12]}$ while in Zambia CHWs were found to provide prompt and effective malaria case management with high adherence. ${ }^{[13]}$ A study from Bangladesh, where CHWs screened villagers, collected sputum samples and administered directly observed therapy for TB treatment, reported high rates of case detection and treatment compliance, with a cure rate of at least $85 \%$ and a drop-out rate of only $3.1 \%{ }^{[14]}$

Again, the success of these programmes depends on continuous training, simple guidelines with standardised protocols, appropriate support and supervision, strong relationships with formal health services and, finally, adequate remuneration or professional development to retain CHWs in the programme. Inadequate remuneration and lack of quality supervision and continuous training are of particular concern and may lead to a decrease in the quality of the programme over time.

While results from ART programmes are promising, limited human resources continue to hamper rollout. Task shifting offers high-quality, cost-effective care to more patients than a physiciancentred model and should be considered for implementation where human resource shortages threaten rollout of programmes. Given the shortfall in healthcare workers there is emerging consensus that some form of NIMART or ART provision by non-physicians will be required to achieve optimal ART coverage in SA. An integrated care model, perhaps in the hands of nurses, which incorporates appropriate management of HIV and TB with non-communicable diseases such as hypertension, diabetes and cancer, could be instrumental in the success of the proposed National Health Insurance scheme. NIMART and other strategies of integrated HIV care may help to alleviate the burden at hospital-based HIV clinics. With approximately 383 nurses per 100000 people in SA (2011 figures), an inclusive approach of community-based organisations, home-based care or even caregivers themselves being able to access treatment may be essential for longterm treatment programme sustainability. While these options are 
likely to be feasible for stable patients, there will always be a need for referral facilities or specialty clinics for particular groups such as children, adolescents, pregnant women or complicated or resistant medical cases.

\section{Evans}

Health Economics and Epidemiology Research Office, Department of Internal Medicine, School of Clinical Medicine, University of the Witwatersrand, Johannesburg, South Africa

Corresponding author: devans@witshealth.co.za

1. Johnson LF. Access to antiretroviral treatment in South Africa, 2004-2011. Southern African Journal of HIV Medicine 2012:13(1):1-8.

2. Herbst AJ, Cooke GS, Barnighausen T, et al. Adult mortality and antiretroviral treatment roll-out in rural KwaZulu-Natal, South Africa. Bull World Health Organ 2009;87(10):754-762. [http://dx.doi rural KwaZulu-Natal, South
org/10.2471/BLT.08.058982]

3. Nyasulu J, Muchiri E, Mazwi S, Ratshefola M. NIMART rollout to PHC facilities increases access to Nyasulu J, Muchiri E, Mazwi S, Ratshefola M. NIMART rollout to PHC facilities increases access to
antiretrovirals in Johannesburg: An interrupted time series analysis. S Afr Med J 2013;103(4):232-236 antiretrovirals in Johannesburg: An interrupted time
(this issue). [http://dx.doi.org/10.7196/SAMJ.6380]

(this issue). [http://dx.doi.org/10.7196/SAMJ.6380]
Chimbindi N, Bärnighausen T, Newell M-L. An integrated approach to improving the availability and 4. Chimbindi N, Bärnighausen T, Newell M-L. An integrated approach to improving the availability and
utilisation of tuberculosis healthcare in rural South Africa. S Afr Med J 2013;103(4):237-240 (this issue). [http://dx.doi.org/10.7196/SAMJ.6423]
5. Georgeu D, Colvin CJ, Lewin S, et al. Implementing nurse-initiated and managed antiretroviral reatment (NIMART) in South Africa: A qualitative process evaluation of the STRETCH trial. Implementation Science 2012; 7:66. [http://dx.doi.org/10.1186/1748-5908-7-66]

6. Sanne I Orrell C Fox MP Conradie F et al.; CIPRA-SA Study Team. Nurse versus doctor management of HIV-infected patients receiving antiretroviral therapy (CIPRA-SA): A randomised non-inferiority
of trial. Lancet 2010;376(9734):33-40. [http://dx.doi.org/10.1016/S0140-6736(10)60894-X]

7. Long L, Brennan A, Fox MP, et al. Treatment outcomes and cost-effectiveness of shifting management of stable ART patients to nurses in South Africa: An observational cohort. PLoS Med 2011;8(7):e1001055. [http://dx.doi.org/10.1371/journal.pmed.1001055]

8. Fairall L, Bachmann MO, Lombard C, et al. Task shifting of antiretroviral treatment from doctors to primary care nurses in South Africa (STRETCH): A pragmatic, parallel, cluster-randomised trial. Lancet 2012;380(9845):889-898. [http://dx.doi.org/10.1016/S0140-6736(12)60730-2]

9. Callaghan M, Ford N, Schneider H. A systematic review of task-shifting for HIV treatment and care in Africa. Human Resources for Health 2010;8(1):8. [http://dx.doi.org/10.1186/1478-4491-8-8]

10. Hermann K, van Dammel W, Pariyo GW, et al. Community health workers for ART in sub-Saharan Africa: Learning from experience - capitalizing on new opportunities. BMC Human Resources for Health 2009;7(1):31. [http://dx.doi.org/10.1186/1478-4491-7-31]

11. Selke HM, Kimaiyo S, Sidle JE, et al. Task-shifting of antiretroviral delivery from health care workers to persons living with HIV/AIDS: Clinical outcomes of a community-based program in Kenya. J Acquir
perat Immune Defic Syndr 2010;55(4):483-490. [http://dx.doi.org/10.1097/QAI.0b013e3181eb5edb]

12. Bari A, Sadruddin S, Khan A, et al. Community case management of severe pneumonia with oral amoxicillin in children aged 2-59 months in Haripur district, Pakistan: A cluster randomised trial. Lancet 2011;378(9805):1796-1803. [http://dx.doi.org/10.1016/S0140-6736(11)61140-9]

13. Chanda P, Hamainza B, Moonga HB, Chalwe V, Pagnoni F. Community case management of malaria using ACT and RDT in two districts in Zambia: Achieving high adherence to test results using community health workers. Malar J 2011;10(1):158. [http://dx.doi.org/10.1186/1475-2875-10-158]

14. Chowdhury AM, Chowdhury S, Islam MN, Islam A, Vaughan JP. Control of tuberculosis by community health workers in Bangladesh. Lancet 1997;350(9072):169-172. [http://dx.doi.org/10.1016/S01406736(96)11311-8]

S Afr Med J 2013;103(4):229-230. DOI:10.7196/SAMJ.6835 